\title{
Demonstration of Wireless Backhauling over Long-Reach PONs
}

\author{
M. Milosavljevic, M. P. Thakur, P. Kourtessis, J. E. Mitchell, J. M. Senior
}

\begin{abstract}
An IEEE 802.16e-2005 (WiMAX) compliant, longreach passive optical network is demonstrated, focusing on the development of next generation optical access with transparent wireless backhauling. In addition to the extended feeder reach, wavelength band overlay is used to enhance network scalability by maintaining passive splitting in the field and with some design modification at the optical line terminal and remote base station. Radio over fibre is used to minimise network installation and maintenance costs through the use of simple remote radio heads complemented by frequency division multiplexing to address individual base stations. The implementation of overlapping radio cells/sectors is also proposed to provide joint signal processing at wireless user terminals. Experimental measurements confirmed EVMs below $-30 \mathrm{~dB}$ and $-23 \mathrm{~dB}$ downstream and upstream respectively over fibre link lengths of up to $84.6 \mathrm{~km}$. In addition, ACLR measurements demonstrated that a figure of $-45 \mathrm{~dB}$ with $40 \mathrm{MHz}$ subcarrier spacing, as specified by the standard can be readily achieved.
\end{abstract}

Index Terms - Integrated -PON-wireless access, optical fibre networks, Radio-over-Fibre (RoF), wireless interoperability for microwave access (WiMAX).

\section{INTRODUCTION}

$\mathrm{W}$ ith developments in broadband wireless networks, driven primarily by WiMAX [1] and LTE [2], data rates up to $1 \mathrm{Gbit} / \mathrm{s}$ are expected to be supported over next generation radio cells. This situation will unavoidably lead to a higher density of base station deployment and a requirement for increased spectral efficiency. High bandwidth, costeffective backhaul links will be required as a result to connect each of these base stations to a common central office (CO). Since the application of current solutions to wireless backhauling, such as SDH and IP, is anticipated to be expensive to both deploy and operate [3], lower-cost optical infrastructures have been suggested, being also capable of

Manuscript received: 03 October 2011. This work was supported in part by the European Network of Excellence, BONE: Building the Future Optical Network in Europe and ACCORDANCE: A Converged copper-optical-radio OFDMA-based access network with high capacity and flexibility, through the 7th ICT Framework Programme.

M. Milosavljevic (e-mail: m.milosavljevic@ herts.ac.uk), P. Kourtessis and J. M. Senior are with the Optical Networks Group, Science and Technology Research Institute (STRI), University of Hertfordshire, Hatfield, AL10 9AB, United Kingdom.

M. P. Thakur (e-mail: m.thakur@ee.ucl.ac.uk) and J. E. Mitchell are with Department of Electronic and Electrical Engineering, University College London (UCL), London, WC1E 6BT, United Kingdom. allowing sharing of network resources among different operators.

Passive Optical Networks (PONs) can satisfy the requirements for increasingly investigated support of wireless backhauling [3, 4]. However, longer-reach architectures, rather than the currently deployed legacy PONs, are anticipated to centrally connect base stations outside urban areas, leading to noticeable reduction in the number of expensive backhauling links. In addition, radio over fibre (RoF) schemes offer a solution for the centralisation of wireless equipment [5-7] enabling link transparency and lower transmission bandwidth per base station (BS) compared to digital-over-fibre (DoF) approaches $[8,9]$. However, RoF links are typically limited by optical component nonlinearities and fibre chromatic dispersion and are therefore available only for short distances and small number of BSs. In alternative approaches, the provision of dedicated wavelengths to each BS over the PON, have been investigated $[10,11]$ to overcome these limitations while addressing optical beat interference. Nevertheless, the strategies proposed so far could result in high cost and scalability problems with an ever increasing number of wireless cells.

Notably, the transport of WiMAX/LTE signals using RoF techniques over PONs, extended to distances of greater than $20 \mathrm{~km}$, has not been collectively examined. This paper also attempts, for the first time, to determine the adjacent channel leakage ratio (ACLR) performance figures that can severely degrade performance over the wireless channels. ACLR plots have scarcely been drawn for point-to-point connections [12] and to the best of our knowledge have not been investigated for point-to-multipoint, long-reach, FDM transmission links. Evaluations are performed by means of optical single sideband (OSSB) modulation in the network downstream to beat chromatic dispersion for longer standard single mode fibre (SSMF) reach, without using receiver pre-amplification in order to further reduce the deployment costs. In addition to ACLR typical error vector magnitude (EVM) characteristics are also plotted.

The rest of the paper is organised as follows. Section II describes the proposed network architecture outlining its novelty. This is followed by the detailed description of the experimental setup for two scenarios (legacy and long reach PON). Section IV presents the performance evaluation of WiMAX signal formats transmitted over typical and long fibre distances. Finally, Section V provides the concluding remarks. 

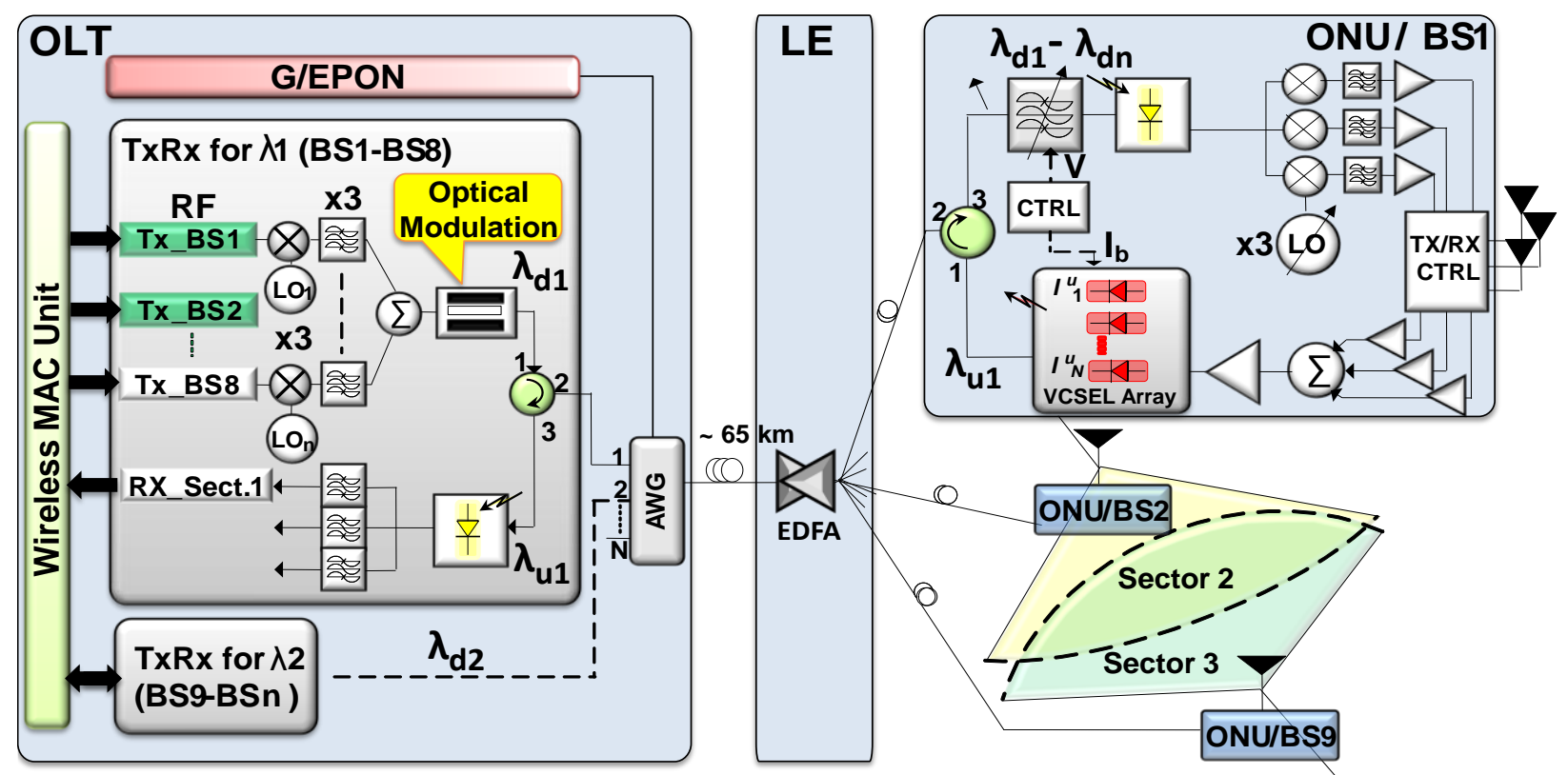

Fig. 1. General description of the proposed architecture for wireless backhauling

\section{KEY NETWORK FEATURES}

For the long-reach topology, shown in Fig. 1, the OLT is based at a $\mathrm{CO}$ in the metro network. At the customer side, optical network unit/base stations (ONU/BSs) are located at either the user premises or at remote antenna posts, providing connectivity to mobile terminals. The proposed network utilizes a local exchange (LE) to connect the OLT through a single $60 \mathrm{~km}$ feeder fibre to multiple users. At the distribution side, the LE is used for broadcasting the downstream (DS) or combining the upstream (US) signals to/from multiple network base stations via a passive splitter and link lengths spans from $20 \mathrm{~km}$ to $40 \mathrm{~km}$ [13]. These features enable node consolidation and a minimal network configuration in the field. Consequently, all radio processing can be located at the OLT resulting in simplified remote base stations. This allows for densely populated base stations to be deployed providing a higher network capacity. For new network deployment the LE can simply be placed at the current OLT and would only perform amplification rather than the legacy OLT functions.

Another significant feature of the aforementioned topology is the attainable scalability with respect to the number of supported BSs, through the application of extended wavelength band overlay. Hence, the passive splitting nature of existing G/EPON infrastructures will be retained. Following the recent ITU standardisation initiatives [14], the 1550-1600 $\mathrm{nm}$ operating window is reserved, as shown in Fig. 2, for RF overlay. To comply with the standard, selected $3.5 \mathrm{GHz}$ WiMAX channels generated in the network OLT are arranged in FDM windows and transmitted over individual wavelengths in the 1550-1600 nm range to selected ONU/BSs. Apart from different wavelengths the proposed architecture also allows for a given FDM window to be propagated on multiple wavelengths. This relaxes the bandwidth requirements of optical and electrical devices while enhancing the network scalability. The resulting reduction in the total FDM bandwidth reduces non-linearity induced penalty by decreasing the optical modulation index (OMI) at the OLT and thus, improves ACLR at remote antennas. The OMI for a laser is defined as:

$$
m_{i}=\frac{\Delta I}{I_{b}-I_{t h}}=\frac{\sqrt{\frac{2 P_{i n}}{R}}}{I_{b}-I_{t h}}=\frac{\alpha}{\beta}
$$

where, $\Delta \mathrm{I}=$ change in the modulating current, $\mathrm{Ib}=$ bias current, $\mathrm{Ith}=$ threshold current, $\mathrm{Pin}=$ average $\mathrm{RF}$ input power and $\mathrm{R}=$ input resistance of the laser.

In practise the application of wavelength band overlay only requires an additional array waveguide grating (AWG) in the OLT and tuneable optical filters in individual ONU/BSs. Importantly the centralised AWG allows for multiple PONs to be serviced by a single OLT while maintaining the distribution network intact in support of smooth migration towards next generation PONs (NG-PONs) [15].

The most notable feature of the proposed network is the introduction of overlapping sectors with centralised control of wireless users at the OLT. This setup uniquely enhances the

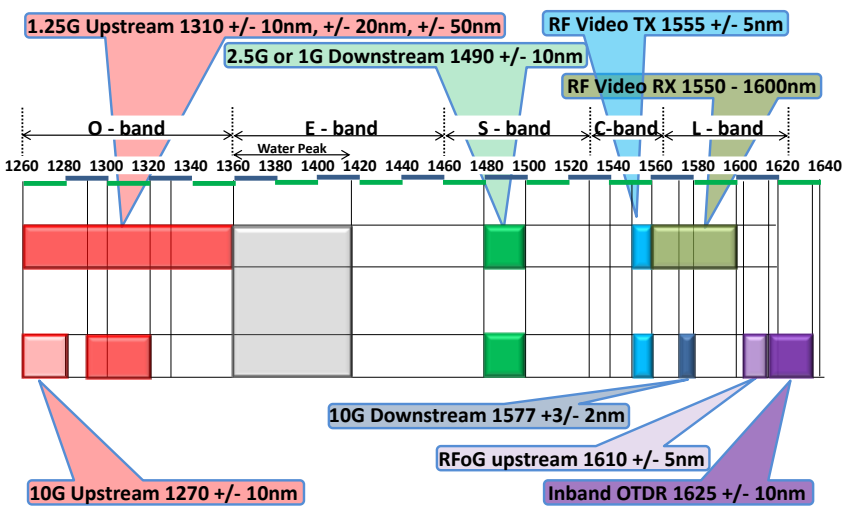

Fig. 2. ITU-T wavelength plan with RF overlay 
joint signal processing and co-ordinated scheduling inherent in traditional WiMAX/LTE deployments. As an example, Fig. 1 portrays centralised rather than independent processing for ONU/BS2 and ONU/BS9. Due to centralised processing, interference mitigation can be greatly improved in future $4 \mathrm{G}$ networks. The latter can be achieved due to substantial reduction of network latency associated with control messages exchanged between base stations. For all sectors a frequency reuse of one is assumed as higher order frequency reuse patterns are practically unproductive.

Another important aspect of this architecture is its agnostic nature in favour of varying wireless signal formats, including LTE that is expected to be widely deployed in the future. To that extent, the outcome of investigations performed in this paper for mobile WiMAX, can be extended to LTE, particularly in the downstream, since they are both based on orthogonal frequency division multiple access (OFDMA).

Finally, to demonstrate colourless wireless-PONs in the upstream direction, long-wavelength vertical cavity surface emitting laser (VCSEL) arrays [16] and tuneable optical bandpass filters (BPF) [17] can be deployed in the ONU/BSs. Although reflective semiconductor optical amplifiers (RSOAs) could be alternatively used, the effects of Rayleigh backscattering are more pronounced in reflective architectures. This is due to re-modulation of downstream carriers for upstream transmission, hence introducing a weak counterpropagating signal [18]. Use of a VCSEL array eliminates this additional penalty. Multi-wavelength propagation in the upstream, with sufficient frequency spacing provided by the VCSEL, also reduces optical beat interference (OBI) at the PIN receiver in the OLT. The use of VCSEL arrays additionally offers simple coupling of optical terminations.

\section{EXPERIMENTAL DEMONSTRATION}

To evaluate the transmission of mobile WiMAX channels over the long-reach PON links between the OLT and ONU/BS, received EVMs were experimentally measured. EVMs give an indication of transmission quality by specifying the minimum acceptable value at each ONU/BS's antenna input. However, for radio transmission over highly nonlinear, power budget limited optical links, EVM performance figures alone are not sufficient. Therefore, ACLR measurements were also performed, in particular for the long reach scenario, in order to establish the adjacent channel leakage power caused by the non-linearity of the optical link for various subcarrier spacing's.

\section{A. IEEE802.16-2005 WiMAX signal generation}

An Aeroflex PXI WiMAX Vector Signal Generator/Analyser unit was used to generate and analyse the WiMAX channels. In particular, the mobile WiMAX 802.16e2005 standard [19] was followed. The specification for each WiMAX channel generated for upstream and downstream transmission is given in Table I.

It is important to stress at this point that, the data rates in Table I were calculated considering guard and pilot subcarriers
TABLE I

IEE802.16E WIMAX TRANSCEIVERS PARAMETERS

\begin{tabular}{lll}
\hline \hline Symbol & Downstream & Upstream \\
\hline FFT size & 1024 & 1024 \\
Modulation & $64-\mathrm{QAM}$ & $16-\mathrm{QAM}$ \\
Coding & $2 / 3$ & $3 / 4$ \\
Bandwidth & $10 \mathrm{MHz}$ & $10 \mathrm{MHz}$ \\
RCE & $-50 \mathrm{~dB}$ & $-25.8 \mathrm{~dB}$ \\
Data rate & $25.2 \mathrm{Mbps}$ & $18.9 \mathrm{Mbps}$ \\
\hline
\end{tabular}

which do not carry user data but rather are employed for synchronisation and estimation purposes. A worst case scenario, $25 \%$ cyclic prefix was also included. The downstream WiMAX channel transmitter relative constellation error ( $\mathrm{RCE}$ ) was $-50 \mathrm{~dB}$, a figure higher than the minimum required for 64-QAM modulation [19]. Finally, the time division duplex (TDD) mode of operation [19] was adopted in the experiment with frame duration of $5 \mathrm{~ms}$.

\section{B. $x P O N$ link demonstration}

Four WiMAX channels forming an FDM window at the standard $3.5 \mathrm{GHz}$ with $30 \mathrm{MHz}$ subcarrier spacing were generated at the OLT, as shown in Fig. 3. In a practical implementation, the number of generated WiMAX channels should match the passive optical network split. Hence, for a targeted 1:16 split to comply with current deployments of legacy PONs, a minimum of 16 channels should be supported. As a proof of concept demonstrator, 4 channels were used to study the system viability. It was also assumed that one or more ONU/BSs would require more than one WiMAX channel. Furthermore, the $30 \mathrm{MHz}$ subcarrier spacing was chosen to match the receiver filter specifications.

To address individual ONU/BSs, each WiMAX channel was shifted in frequency from $3.5 \mathrm{GHz}$ to a higher value that avoids adjacent channel interference. The 4 channels were upconverted to $3.955 \mathrm{GHz}, 3.985 \mathrm{GHz}, 4.015 \mathrm{GHz}$ and 4.045 $\mathrm{GHz}$. This was achieved by using a predetermined local oscillator (LO) and BPFs in the OLT. The WiMAX channels were then combined and modulated onto an optical carrier. At the ONU/BS, an LO was required, operating at exactly the same frequency for a specific ONU/BS to downshift the appropriate WiMAX channels. Multiple BPFs were also needed to select each channel prior to transmission over the air channel.

As the fibre chromatic dispersion induced penalty for $20 \mathrm{~km}$ distances is tolerable, optical double sideband modulation (ODSB) was used for XPON tests. The FDM formed signals at the OLT were optically transmitted using a Mach-Zehnder modulator (MZM) and a commercially available distributed feedback (DFB) laser source. The MZM offered improved performance in terms of chirp compared to direct modulation but at a higher cost. However, as it is typically shared by large number of users, cost is not a limiting factor.

The resulting optical signal on $\lambda_{\mathrm{d} 1}=1558.2 \mathrm{~nm}$, was transmitted through a circulator and a $16 \times 1$ AWG to an ONU/BS, using SSMF ranging from $23.2 \mathrm{~km}$ to $40.7 \mathrm{~km}$. The various optical component losses in the system's OLT, 


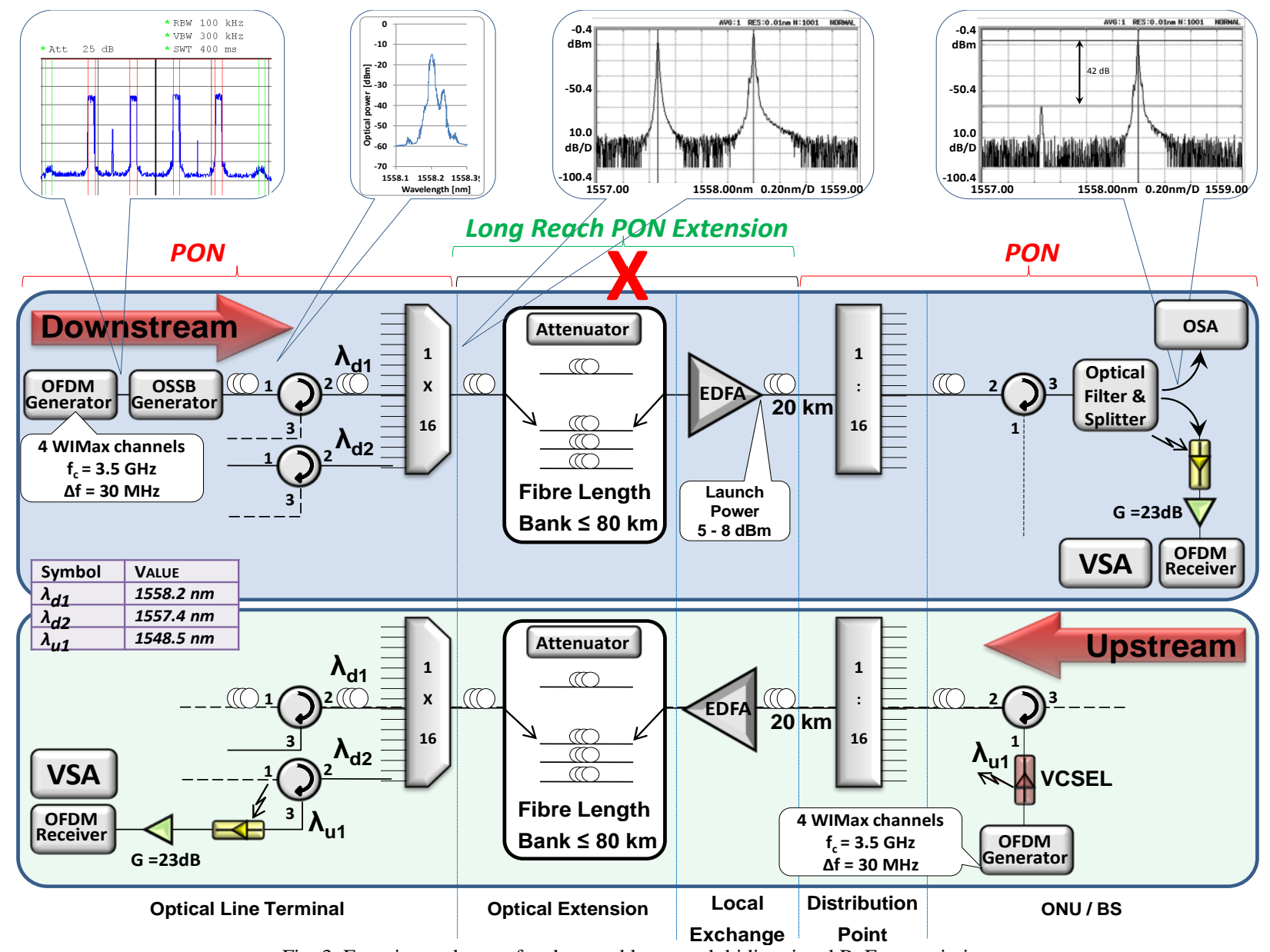

Fig. 3. Experimental setup for short and long reach bidirectional RoF transmission

provided in Table II, meant that the launched optical power into the fibre link was $0.9 \mathrm{dBm}$. An additional unmodulated signal at wavelength $\lambda_{\mathrm{d} 2}=1557.4 \mathrm{~nm}$ was transmitted through the AWG to determine the interference at ONU/BSs.

Quite importantly, if there was a requirement to accommodate legacy PONs over the architecture for smooth network migration, this could be achieved by utilising the free spectral range of the AWG allowing 1490/1310 nm to be transmitted.

TABLE II

OPTICAL LOSSES

\begin{tabular}{ll}
\hline \hline Symbol & Loss (dB) \\
\hline MZM & 7.75 \\
AWG & 3.76 \\
Circulator & 0.9 \\
Pol. Control & 0.45 \\
Splitter $(1: 16)$ & 13.5 \\
Add/Drop filter & 5 \\
\hline
\end{tabular}

Continuing in the downstream an optical attenuator was used after the fibre link to account for varying optical splits (1: $\mathrm{X})$. At the ONU/BS, an add/drop multiplexer (ADM) was used prior to PIN detection as a substitute for a commercial 100 $\mathrm{GHz}$ optical band pass filter, displaying the same pass-band characteristics. The ADM drop port, at the wavelength of $1558.2 \mathrm{~nm}$, produced a signal to interference ratio of $42 \mathrm{~dB}$. The resulting up-converted WiMAX signal was then down- shifted in frequency to get the original WiMAX channels and subsequently amplified by a $23 \mathrm{~dB}$ gain amplifier for performance evaluation measurements.

A single IEEE 802.16-2005, $3.5 \mathrm{GHz}$ channel was generated for the upstream to directly modulate a VCSEL at $\lambda_{\mathrm{u} 1}=1548.5 \mathrm{~nm}, 8.3 \mathrm{~mA}$ bias and $-0.94 \mathrm{dBm}$ launch power. As the demonstration involves no signal transmission over a wireless channel, the upstream WiMAX transmitter RCE was set to $-25.8 \mathrm{~dB}$ to conform to the figure expected to be received at the base station in a practical scenario. The resulting optical signal was then routed through the corresponding AWG output port to the destination receivers (Rx_Sect.X) in the OLT where individual EVMs were measured.

\section{Long-Reach PON demonstration}

The experimental set-up expansion to accommodate longreach networking is also shown in Fig. 3. The 40-80km feeder extension was of SSMF type, added to the xPON (as in subsection B) with a multi-wavelength overlay being provided over an already deployed passive splitter.

A variable-gain erbium doped fibre amplifier (EDFA) was used to compensate for the increased link losses, located in the LE where the feeder fibre terminates. The application of EDFAs is widely accepted for NG-PONs by operators and vendors [15]. The EDFA utilised for the demonstration has a 
noise figure of $6 \mathrm{~dB}$ and variable gain up to $28 \mathrm{~dB}$.

Unlike the ODSB used in the previous section, OSSB modulation was used in the downstream to mitigate the penalty due to chromatic dispersion in long-reach PON spans. OSSB is generated using a dual arm MZM, to achieve significant cost reduction compared to alternative solutions based on optical band pass filters [20]. WiMAX channels, specified in Table I, were fed to the MZM with a 90 degree phase shift between the two MZM arms. The resulting optical spectrum is shown as an inset in Fig. 3.

To complete the downstream transmission, the output of the MZM was then fed to the optical circulator and an AWG as described in subsection B. Power budget optimisation at varying feeder fibre link spans was performed to determine the maximum transmission distance as well as the optimum performance in terms of ACLR and EVM. The length of the distribution network from the LE to the ONU was maintained fixed at $20 \mathrm{~km}$, complying with typical next generation access network targets for long-reach networks. The ONU/BS premises infrastructure was also unchanged with the network upstream still utilising double sideband direct modulation of the VCSEL.

\section{RESULTS AND DISCUSSIONS}

\section{A. $20 \mathrm{~km}$ PON WiMAX transmission}

Of the four frequency-shifted, $3.5 \mathrm{GHz}$, WiMAX channels, each displayed a $50 \mathrm{~dB}$ signal to noise ratio as specified by the Aeroflex PXI transmitter. In order to measure intermodulation distortion and evaluate the associated degradation imposed on the received WiMAX channels at the ONU/BS receivers, the Aeroflex output power was varied between $-2 \mathrm{dBm}$ and +14 $\mathrm{dBm}$.

The EVM characteristic of a selected WiMAX channel as a function of the MZM $(\mathrm{V} \pi=2.56 \mathrm{~V}) \mathrm{RF}$ drive power was measured at the remote antenna input of the corresponding ONU/BS as shown in Fig. 4, for varying fibre link lengths and network splits to define the maximum reach and splitting ratio attainable with a fully passive infrastructure. At low RF drive powers the dominant signal impairment is noise. However, at high RF powers, EVM increases due to the nonlinearities of the MZM. To comply with the WiMAX downstream requirements for 64-QAM 2/3 [19], an EVM figure above -30 $\mathrm{dB}$ needs to be achieved at the bottom of the bathtub curve. This was obtained in the demonstration for a $23.2 \mathrm{~km}$ fibre link and 16 split with RF input powers into the MZM ranging between $+11 \mathrm{dBm}$ and $+13 \mathrm{dBm}$ and a moderate $+1 \mathrm{dBm}$ optical power launched into the fibre.

Maintaining the MZM OMI and optical launch power at the bottom of the bathtub curve to rule out noise and non-linearity induced penalty, further tests were carried out to assess link performance for various split ratios and fibre lengths. As shown in Fig. 4, reducing the split ratio, e.g. $40.7 \mathrm{~km}$ and 8split point, the link performance is improved $(\mathrm{EVM}=-31 \mathrm{~dB})$. Hence, it can be concluded that a justifiable increase in the fibre launch power would allow higher length-split ratios. This is expected to be achieved in a practical network by the optimisation of OLT network component losses. Similar results were confirmed for all WiMAX channels.

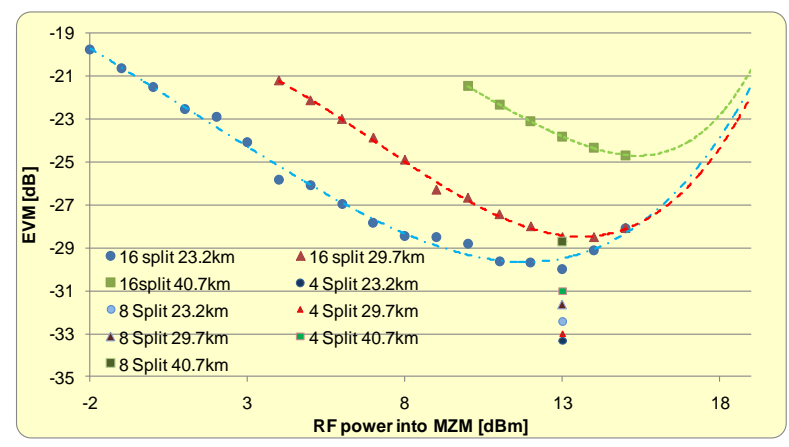

Fig. 4. EVM versus MZM RF power at remote antenna downstream.

EVM measurements were also taken at the OLT receiver in the upstream in order to establish the signal degradation between the base station and the OLT and these are shown in Fig. 5, as a function of the VCSEL $\left(\mathrm{I}_{\mathrm{th}}=1.7 \mathrm{~mA}, \mathrm{I}_{\text {bias }}=7 \mathrm{~mA}\right)$ $\mathrm{RF}$ input power. An EVM of $-23 \mathrm{~dB}$ was recorded over 23.2 $\mathrm{km}$ of fibre with 16 split. The interaction of VCSEL laser chirp and fibre dispersion in the upstream analogue optical modulation had negligible effect on the received WiMAX channels in the OLT. Longer fibre link lengths were not used due to the VCSEL output power limitation (max OdBm); however, higher output power VCSEL arrays [16] could significantly reduce the EVM penalties.

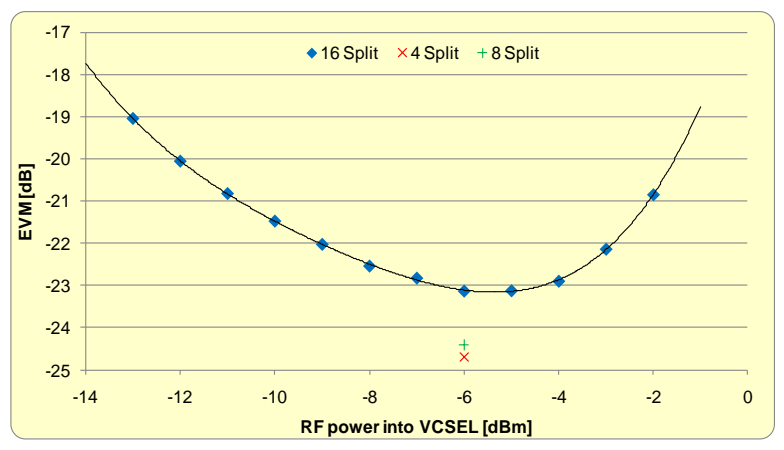

Fig. 5. EVM versus MZM RF power at the OLT upstream

Finally, the power of the received downstream WiMAX signals at an ONU/BS were determined to be approximately $30 \mathrm{dBm}$ and within the range of input powers required for linear operation of the high power amplifier for transmission over the air channel. Also, the constellation diagrams obtained after phase and amplitude corrections at the remote antenna are displayed in Fig. 6. It should be noted that the two outermost points represent the pilot tones used for estimation and synchronisation purposes.

\section{B. WiMAX signal routing over $L R-P O N$ s}

The key measurement for long reach network performance was the subcarrier spacing needed to sustain acceptable ACLR values. Only downstream measurements were considered because, for RoF designs, it is imperative that the ACLR before transmission into air conforms to regulatory requirements. In the upstream, the received channels are 
terminated at the OLT, so ACLR degradation can be compensated.

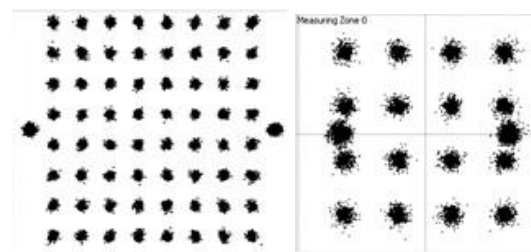

Fig. 6. 64-QAM downstream (left) and 16-QAM upstream (right)

The ACLR is dependent on several factors including the length of the transmission link, OMI, subcarrier spacing and the number of subcarriers. The same four, frequency shifted WiMAX channels employed in Section III were utilised. The spacing between WiMAX channels was varied between 10 $\mathrm{MHz}$ and $40 \mathrm{MHz}$ (in $5 \mathrm{MHz}$ increment), as shown in Fig. 7.

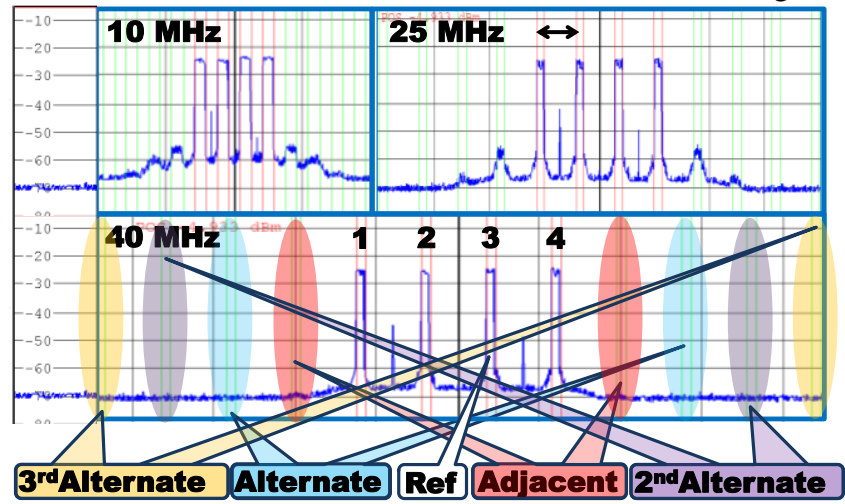

Fig. 7. WiMAX channels spacing and ACLR components

A feeder fibre length of $65 \mathrm{~km}$, with an EDFA at the LE, was used for these measurements with a distribution network of $20 \mathrm{~km}$ as described in Section III. The gain of the EDFA was varied to adjust the optical launch power from $5 \mathrm{dBm}$ to 9 $\mathrm{dBm}$ into the distribution and feeder fibre links in order to determine the transmission performance. The channel spacing versus measured ACLR with respect to the third WiMAX channel is shown in Fig. 8. The specific channel selected represents the worst case scenario in terms of both adjacent

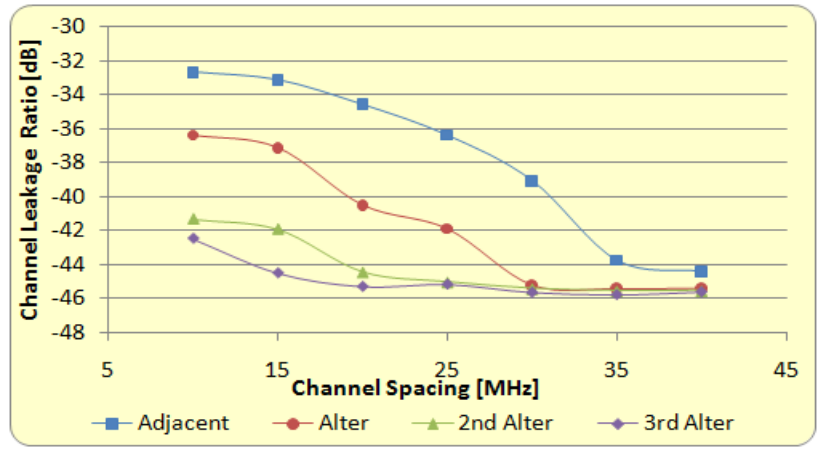

Fig. 8. ACLR versus WiMAX channels spacing

and alternate channel interference as described in Fig. 7. For mobile WiMAX applications the acceptable ACLR figure is usually specified by local regulators and for $10 \mathrm{MHz}$ bandwidth, $-35 \mathrm{~dB}$ for adjacent and $-45 \mathrm{~dB}$ for alternate channels is required to be achieved [21]. It may be observed from Fig. 8 that for $40 \mathrm{MHz}$ subcarrier spaced FDM windows both adjacent and alternate channels satisfy the requirements.
At $20 \mathrm{MHz}$, although the ACLR condition for adjacent channels is compliant with the standard, the alternate channels fall above the $-45 \mathrm{~dB}$ threshold. This performance however does not create a network limitation because $40 \mathrm{MHz}$ subcarrier spacing for FDM applications is still a low bandwidth requirement for the PON infrastructure. To illustrate the above point, assuming 16 base stations, $10 \mathrm{MHz}$ WiMAX bandwidth and $40 \mathrm{MHz}$ FDM subcarriers spacing, the total backhauling bandwidth requirement will be $640 \mathrm{MHz}$ $(16 \times 40 \mathrm{MHz})$ which indicates that low cost optical and electrical components could be used for network implementation.

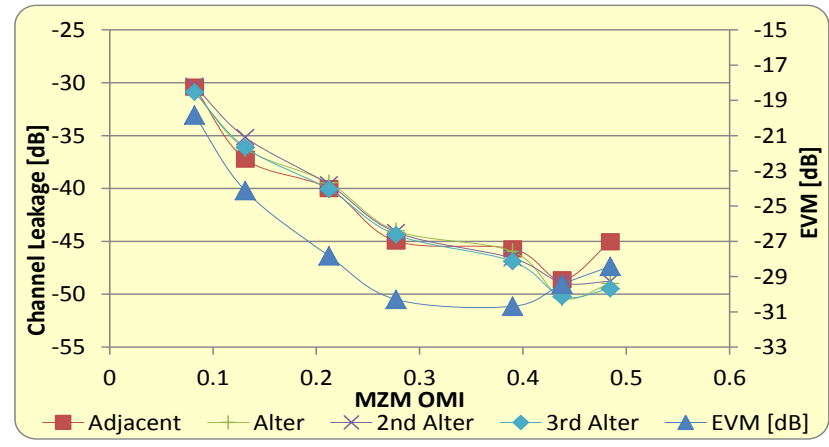

Fig. 9. ACLR versus EVM for different MZM OMI

Fig. 9 displays the EVM obtained at an antenna input with respect to MZM OMI and ACLR. The characteristic at the bottom of the bathtub curve confirms that an EVM of $-30 \mathrm{~dB}$ was obtained as required for the standard [19] for $65 \mathrm{~km}$ feeder. From the plot, it can also be inferred that the specified OMI range meets the adjacent and alternate channels ACLR profile of $-45 \mathrm{~dB}$, as shown in Fig. 8 for the $40 \mathrm{MHz}$ subcarrier spacing.

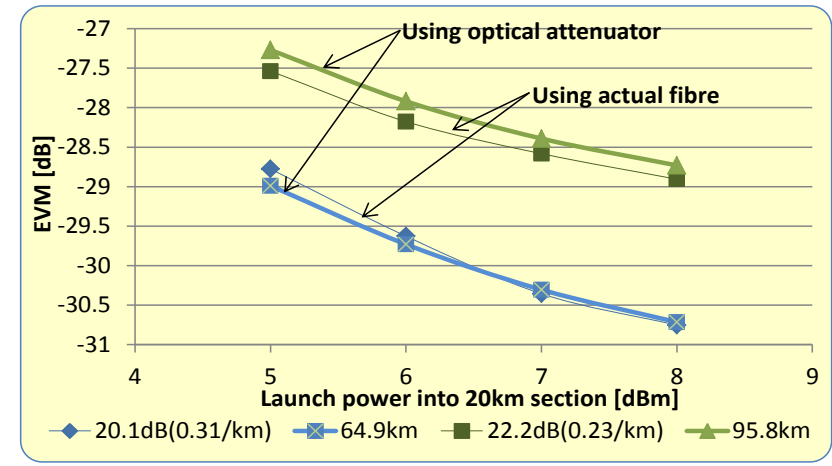

Fig. 10. EVM with fibre and equivalent attenuation figures downstream

To ascertain the chromatic dispersion penalty on EVM, measurements with and without the feeder were compared with the corresponding attenuation using a variable attenuator. As shown in Fig. 10 the imposed degradation in downstream as a result of chromatic dispersion for various LE output powers is negligible. This is due to OSSB implementation for the extended $65 \mathrm{~km}$ feeder reach. For an EVM of $-30 \mathrm{~dB}$ the minimum required optical output power is $+6.5 \mathrm{dBm}$. This was achieved with an EDFA gain of $20 \mathrm{~dB}$.

The fibre dispersion accumulation in EVMs measured at the OLT for upstream is plotted in Fig. 11, for a range of LE output powers. 


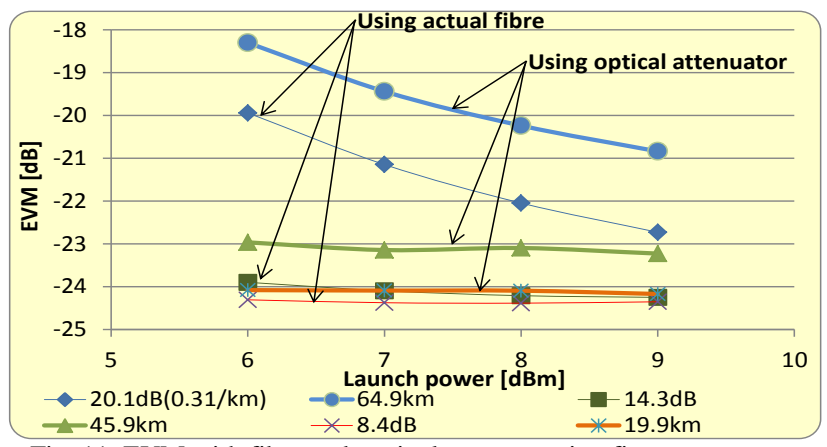

Fig. 11. EVM with fibre and equivalent attenuation figures upstream

A power penalty of $1.8 \mathrm{~dB}$ may be observed for the same transmission distance. This is due to ODSB implementation in the upstream direction. As a result it restricts feeder transmission to $45 \mathrm{~km}$, and therefore adding the typical $20 \mathrm{~km}$ distribution fibre, a total of $65 \mathrm{~km}$ upstream backhauling links can be achieved. In order to obtain symmetrical links in downstream and upstream, OSSB modulation can be implemented for upstream with an additional optical filter in the ONU/BS to select single sideband.

\section{CONCLUSIONS}

A novel wireless/optical access network architecture has been demonstrated, providing high capacity backhauling links for mobile WiMAX. Emerging broadband wireless standards such as LTE can also be supported due to the architecture's transparency to wireless formats. The network exhibits FDM for ONU/BS selectivity, RoF providing for a low bandwidth requirement in the optical domain, long reach fibre spans utilising OSSB modulation, power-splitting to maintain legacy deployments in the field and wavelength band overlay to comply with NG-PON deployment for service overlay. The extended wavelength band overlay also translates to reduced component costs, as the same FDM windows can be utilized on multiple-wavelength signals requiring relatively low bandwidth optical and electrical devices. This in turn improves ACLR and lowers OMI due to a decrease in the penalty imposed by the non-linearity of components. The targeted ACLR of $-45 \mathrm{~dB}$ for $84.6 \mathrm{~km}$ downstream transmission was fully demonstrated with maximum subcarrier spacing of 40 $\mathrm{MHz}$ and with cost effective OSSB generation. In addition, IEEE 802.16-2005 compliance was achieved with EVM figures below $-30 \mathrm{~dB}$. Long wavelength, low cost VCSEL arrays for upstream also demonstrated - $23 \mathrm{~dB}$ EVMs over a 65 $\mathrm{km}$ transmission distances with ODSB modulation. Hence, RoF complemented with FDM provides for resource sharing of the same infrastructure between different operators which is considered as a prerequisite for next generation wireless backhauling.

\section{REFERENCES}

[1] S. Ahmadi, "An overview of next-generation mobile WiMAX technology," IEEE Communications Magazine, vol. 47, pp. 84-98, (2009).
[2] Evolved Universal Terrestrial Radio Access (E-UTRA): LTE Physical Layer - General description, 3GPP TS 36.201, 2008

[3] ACCORDANCE (2010, November 20). ACCORDANCE: A Converged Copper-Optical-Radio OFDMA-based access Network with high Capacity and Flexibility [Online]. Available: http://ict-accordance.eu

[4] M. Milosavljevic, P. Kourtessis, and J. M. Senior, "Multi-Wavelength WiMAX-PONs With Overlapping Cells," J. Opt. Commun. Netw., vol. 3, pp. 172-177, (2011).

[5] J. Zhensheng, Y. Jianjun, G. Ellinas, and G. K. Chang, "Key Enabling Technologies for Optical-Wireless Networks: Optical Millimeter-Wave Generation, Wavelength Reuse, and Architecture," J. Lightw. Technol., vol. 25, pp. 3452-3471, (2007).

[6] M. G. Larrode, A. M. J. Koonen, J. J. V. Olmos, and A. Ng'Oma, "Bidirectional radio-over-fiber link employing optical frequency multiplication," Photon. Technol. Lett., vol. 18, pp. 241-243, (2006).

[7] F. Lecoche, E. Tanguy, B. Charbonnier, H. Li, F. van Dijk, A. Enard, F. Blache, M. Goix, and F. Mallecot, "Transmission Quality Measurement of Two Types of $60 \mathrm{GHz}$ Millimeter-Wave Generation and Distribution Systems," J. Lightw. Technol., vol. 27, pp. 5469-5474, (2009).

[8] Y. Kun, O. Shumao, K. Guild, and C. Hsiao-Hwa, "Convergence of ethernet PON and IEEE 802.16 broadband access networks and its QoSaware dynamic bandwidth allocation scheme," J. Select. Areas Commun., vol. 27, pp. 101-116, (2009).

[9] M. Milosavljevic, P. Kourtessis, and J. M. Senior, "Wireless Convergence over Next Generation OFDMA-PONs," presented at Access Networks and In-house Communications (ANIC), OSA Technical Digest (CD), (Optical Society of America, 2010), paper ATuB3, 2011

[10] S. Wei-Tao, W. Shing-Wa, C. Ning, K. Balasubramanian, Z. Xiaoqing, M. Maier, and L. G. Kazovsky, "Hybrid Architecture and Integrated Routing in a Scalable Optical-Wireless Access Network," J. Lightw. Technol., vol. 25, pp. 3443-3451, (2007).

[11] N. Ghazisaidi, M. Maier, and C. Assi, "Fiber-wireless (FiWi) access networks: a survey," IEEE Communications Magazine, vol. 47, pp. 160167, (2009).

[12] F. Florian, C. Benoit, and A. Catherine, "3GPP Compliant Downlink ACLR Performances Of PON Distributed Multiple UMTS FDD Carriers," presented at Access Networks and In-house Communications, 2010.

[13] Gigabit-capable passive optical networks (GPON): Physical media dependent (PMD) layer specification, ITU-T G.984.2, 2003

[14] K. Tanaka, A. Agata, and Y. Horiuchi, "IEEE 802.3av 10G-EPON Standardization and Its Research and Development Status," J. Lightw. Technol., vol. 28, pp. 651-661, (2010).

[15] J. i. Kani, F. Bourgart, A. Cui, A. Rafel, M. Campbell, R. Davey, and S. Rodrigues, "Next-generation PON-part I: technology roadmap and general requirements," IEEE Communications Magazine, vol. 47, pp. 43-49, (2009).

[16] W. Hofmann, E. Wong, G. Bohm, M. Ortsiefer, N. H. Zhu, and M. C. Amann, "1.55-um VCSEL Arrays for High-Bandwidth WDM-PONs," Photon. Technol. Lett., vol. 20, pp. 291-293, (2008).

[17] M. Maier, "WDM Passive Optical Networks and Beyond: the Road Ahead (Invited)," IEEE/OSA J. Opt. Commun. Netw., vol. 1, pp. C1C16, (2009).

[18] B. Schrenk, G. de Valicourt, J. A. Lazaro, R. Brenot, and J. Prat, "Rayleigh Scattering Tolerant PON Assisted by Four-Wave Mixing in SOA-based ONUs," J. Lightw. Technol., vol. 28, pp. 3364 - 3371, (2010).

[19] "IEEE Standard for Local and metropolitan area networks Part 16: Air Interface for Fixed and Mobile Broadband Wireless Access Systems Amendment 2: Physical and Medium Access Control Layers for Combined Fixed and Mobile Operation in Licensed Bands and Corrigendum 1," IEEE Std 802.16e-2005 and IEEE Std 802.162004/Cor 1-2005 (Amendment and Corrigendum to IEEE Std 802.162004), pp. 0_1-822, (2006).

[20] H.-H. Lu, W.-S. Tsai, H.-C. Peng, and Y.-J. Ji;, "A comparison between optical SSB transmitter-filter in a full-duplex radio-on-fiber transport system," IEEE Communications Letter, vol. 9, pp. 649-651, (2005).

[21] Anritsu. (2011, July 10). Mobile WiMAX Base Station Troubleshooting Guide [Online]. Available: http://www.anritsu.com 